\title{
ARTRODESE LOMBAR MINIMAMENTE INVASIVA COM ACESSO INTERMUSCULAR SEM MATERIAL CIRÚRGICO ESPECIAL: ESTUDO DE SÉRIE DE CASOS
}

\author{
MINIMALLY INVASIVE LUMBAR ARTHRODESIS WITH INTERMUSCULAR APPROACH \\ WITHOUT SPECIAL SURGICAL MATERIAL: A CASE SERIES \\ ARTRODESIS LUMBAR MÍNIMAMENTE INVASIVA CON ACCESO INTERMUSCULAR SIN \\ MATERIAL QUIRÚRGICO ESPECIAL: ESTUDIO DE SERIE DE CASOS
}

Bruno de Azevedo Oliveira', Marcelo Simoni Simöes², Ernani Vianna Abreu³

\begin{abstract}
RESUMO
Objetivos: Analisar os resultados clínicos de uma série de pacientes com doença degenerativa da coluna lombar tratados com artrodese circunferencial com acesso minimamente invasivo intermuscular sem material cirúrgico especial. Métodos: Análise de uma série prospectiva de 12 pacientes consecutivos não-randomizados submetidos à fusão lombossacra de 1 nível para doença degenerativa. Avaliados os Índices de Oswestry 2.0 e a escala visual analógica de dor (VAS) no pré-operatório e seis meses após a cirurgia. A artrodese foi realizada por acesso paramediano bilateral entre os músculos multifidus e longissimus com o uso de afastador cervical simples com lâminas cambiáveis e implantes convencionais. Resultados: Houve uma melhora média de 3,6 pontos na VAS e 27,5 pontos percentuais no Índice de Oswestry quando comparadas as avaliações pré-operatórias e após seis meses de follow-up. As melhoras mais marcadas foram nos pacientes que apresentavam ciatalgia por hérnia discal associada à discopatia. Os quesitos do Índice de Oswestry que apresentaram melhor resultado foram a intensidade da dor e a qualidade do sono. Os que apresentaram pior resultado foram a capacidade de levantamento de pesos e a dor ao sentar. Não houve dificuldade adicional devido à técnica e ao material utilizado. Conclusões: A artrodese da coluna lombossacra por abordagem minimamente invasiva transmuscular pode ser realizada com afastadores cirúrgicos normais e implantes semelhantes ao da técnica tradicional sem prejuízo técnico ou no resultado clínico.
\end{abstract}

Descritores: Procedimentos cirúrgicos minimamente invasivos; Coluna vertebral; Artrodese; Dor lombar.

\begin{abstract}
Objectives: To analyze the clinical results of a series of patients with degenerative disease of the lumbar spine treated with circumferential arthrodesis with minimally invasive intermuscular approach without special surgical material. Methods: Analysis of a prospective series of 12 consecutive non-randomized patients undergoing single level lumbosacral fusion for degenerative disease. Oswestry Disability Index (ODI version 2.0) and visual analogue pain scale (VAS) were performed preoperatively and six months after surgery. Arthrodesis was performed with bilateral paramedian approach between the multifidus and longissimus muscles using simple cervical retractor systems and conventional implants. Results: There was an average improvement of 3.6 points on the VAS and 27.5 percentage points in the ODI comparing the evaluations performed preoperatively and six months after surgery. The improvement was higher in patients with sciatica by disc herniation associated with discopathy. There was no additional difficulty related to the technique and surgical material used. Conclusions: Circumferential lumbosacral arthrodesis with minimally-invasive intermuscular approach can be performed with normal surgical retractors and conventional implants without compromising the clinical outcome on patients with degenerative disease of the spine.
\end{abstract}

Keywords: Surgical procedures, minimally invasive; Spine; Arthrodesis; Low back pain.

\section{RESUMEN}

Objetivos: Analizar los resultados clínicos de una serie de pacientes con enfermedad degenerativa de la columna lumbar, tratados con artrodesis circunferencial con acceso intermuscular mínimamente invasivo, sin material quirúrgico especial. Métodos: Análisis de una serie prospectiva de 12 pacientes consecutivos, no seleccionados aleatoriamente, sometidos a la fusión de un sólo nivel lumbosacro por enfermedad degenerativa. El Índice de Discapacidad de Oswestry (ODI versión 2.0) y la escala visual analógica de dolor (VAS) fueron analizados antes de la cirugía y 6 meses después. La artrodesis se realizó con el acceso paramediano bilateral entre los músculos multifidus y longisimus utilizando sistemas simples de retractores cervicales, con láminas sustituibles, e implantes convencionales. Resultados: Se observó una mejoría media de 3,6 puntos en la VAS y 27,5 puntos porcentuales en el ODI comparado con las evaluaciones realizadas antes y 6 meses después de la cirugía. La mejora fue mayor en los pacientes con ciática por hernia discal asociada con discopatía. Las preguntas del Indice de Oswestry, que presentaron mejores resultados, fueron la intensidad del dolor y la calidad del sueño. Las que tuvieron peores resultados fueron la capacidad de levantamiento de pesos y el dolor al sentarse. No hubo mayores dificultades relacionadas con la técnica y el material utilizado. Conclusiones: La artrodesis de la columna lumbosacra, con abordaje intermuscular mínimamente invasivo, es posible de ser realizada con retractores quirúrgicos normales e implantes semejantes a los de la técnica tradicional, sin perjuicio técnico y sin comprometer el resultado clínico .

\section{Descriptores: Procedimientos quirúrgicos mínimamente invasivos; Columna vertebral; Artrodesis; Dolor de la región lumbar}

\footnotetext{
1 - Neurocirurgião Professor Auxiliar do Departamento de Clínica Cirúrgica da Universidade Estadual de Londrina - Londrina (PR), Brasil.

2 - Neurocirurgião do Serviço de Cirurgia de Coluna do Hospital Ernesto Dornelles. Mestre em Engenharia eTecnologia de Materiais pela PUC-RS - Porto Alegre (RS), Brasil.

3 - Ortopedista do Serviço de Cirurgia de Coluna do Hospital Ernesto Dornelles - Porto Alegre (RS), Brasil.
} 


\section{INTRODUÇÃO}

Os acessos minimamente invasivos foram desenvolvidos com intuito de reduzir o trauma cirúrgico, preservando a anatomia e, por consequência, reduzindo a dor pós-operatória e o tempo de internação, permitindo mobilização precoce, recuperação mais rápida e custos menores ${ }^{1}$.

Vários estudos de abordagens minimamente invasivas relataram resultados clínicos melhores, em comparação com a técnica tradicional ${ }^{2,3}$ embora com insuficiente estudo estatístico

Um dos problemas para a difusão destas técnicas é que, embora elas dependam apenas de instrumental que permita uma abordagem cirúrgica restrita, as grandes empresas do ramo costumam associá-las ao uso de implantes de alto custo, o que, por culpa do contexto socioeconômico em que trabalhamos, de certa forma impede a sua aplicação e difusão.

Foi realizado o estudo de uma série de 12 pacientes com doença degenerativa da coluna vertebral tratados com artrodese intersomática minimamente invasiva por via posterior intermuscular, utilizando um conjunto de afastadores cirúrgicos para coluna cervical, com implantes intersomáticos e sistemas pediculares convencionais, de diferentes fabricantes, e analisado os resultados clínicos com período de seguimento mínimo de 6 meses.

\section{MATERIAIS E MÉTODOS}

Foi analisada uma série prospectiva de 12 pacientes não randomizados, submetidos a cirurgia de artrodese da coluna lombossacral de 1 nível para o tratamento de doenças da coluna lombossacra. Foram incluídos pacientes com dor lombar ou lombociática resistente ao tratamento conservador e relacionada à presença de lesão apenas nos segmentos L4-5 ou L5-S1. As doenças foram discopatia degenerativa com ou sem hérnia discal associada e espondilolistese espondilolítica com degeneração discal (Tabela 1). O critério de exclusão foi a presença de obesidade (IMC > 30). Os pacientes foram avaliados no pré-operatório e 6 meses após a cirurgia pela escala de Oswestry 2.0 e pela escala visual analógica de dor (VAS).

Tabela 1. Resumo dos casos e indicações.

\begin{tabular}{|c|c|c|c|c|}
\hline Paciente & Sexo & Idade & Indicação & Nível \\
\hline 1 & $\mathrm{~F}$ & 37 & Discopatia e hérnia & L5-S1 \\
\hline 2 & M & 22 & Listese lítica & L5-S1 \\
\hline 3 & $\mathrm{~F}$ & 27 & Discopatia e hérnia & L5-S1 \\
\hline 4 & $\mathrm{~F}$ & 47 & Discopatia grave & L5-VT \\
\hline 5 & M & 42 & Discopatia e hérnia pós-discectomia & L4-L5 \\
\hline 6 & $\mathrm{~F}$ & 43 & Listese lítica & L4-L5 \\
\hline 7 & $\mathrm{~F}$ & 39 & Discopatia grave & L5-S1 \\
\hline 8 & M & 26 & Discopatia com hérnia & L5-S1 \\
\hline 9 & M & 48 & Discopatia & L4-L5 \\
\hline 10 & $\mathrm{~F}$ & 46 & Discopatia & L4-L5 \\
\hline 11 & $\mathrm{M}$ & 51 & Discopatia com hérnia & L5-S1 \\
\hline 12 & M & 27 & Discopatia & L5-S1 \\
\hline
\end{tabular}

A artrodese foi realizada através de duas incisões lombares paramedianas com aproximadamente 3 a 4cm de extensão. A localização das incisões foi marcada na pele com radioscopia em anteroposterior verdadeiro, traçando-se uma linha entre as bordas laterais dos pedículos a ser abordados (Figura 1). Após a abertura da fáscia dorsolombar, foi identificado e dissecado o plano entre os músculos longisimus e multifidus, até a identificação da faceta articular. Nesse momento instalou-se um afastador cervical comum com lâminas cambiáveis (Caspar Cervical Retractor System - Aesculap B. Braun, Tutlingen, Alemanha). A abordagem do espaço foi realizada com ressecção da faceta articular inferior da vértebra de cima, seguida de ressecção parcial da faceta superior da vértebra de baixo, expondo-se o disco e raiz nervosa (Figura 2). Dependendo do caso, a facetectomia para acesso ao canal foi realizada de um ou ambos os lados. A indicação primária para abertura bilateral foi compressão radicular bilateral, mas em dois casos a abertura bilateral do canal foi necessária devido ao uso de espaçadores intersomáticos do tipo cage retangular, que não permite colocação de dois implantes pelo mesmo lado. A descompressão e discectomia foram realizadas pelas técnicas tradicionais, com curetas e pinças de disco. A artrodese foi realizada com o enxerto retirado das lâminas e impactado em um par de espaçadores intersomáticos de diferentes modelos e fornecedores. Os espaçadores foram colocados no espaço discal por um ou por ambos os lados, dependendo do acesso uni ou bilateral. Em quatro casos foi retirado enxerto ilíaco pela mesma incisão de acesso. Nos casos em que se realizou facetectomia unilateral, a articulação não ressecada foi aberta e as superfícies articulares escarificadas e impactadas com enxerto ósseo. Após, foi realizada fixação pedicular com parafusos de diferentes modelos e fornecedores. A colocação dos parafusos foi realizada conforme os procedimentos usuais, com localização do ponto de entrada por referenciais anatômicos e preparo do orifício de colocação com puncionador manual e probe (Figura 3). Todo o procedimento foi controlado por radioscopia. Após o fim do procedimento foi realizada sutura da fáscia dorsolombar, subcutâneo e pele. Não foi utilizado nenhum tipo de dreno. O esquema padrão de analgesia pós-operatória foi tramadol e dipirona endovenosos a cada 6 horas, além de um esquema de resgate com morfina endovenosa até de 1 em 1 hora. A analgesia foi reduzida gradualmente, conforme tolerância do paciente. A fisioterapia foi iniciada e os pacientes foram liberados para deambulação a partir do primeiro $\mathrm{PO}$, assim que estivessem dispostos a sair do leito. O critério de alta foi uso de analgesia oral e autonomia para tarefas básicas da vida diária, como levantar, ir ao banheiro e alimentar-se de forma independente. Fisioterapia ambulatorial foi mantida por no mínimo 60 dias.
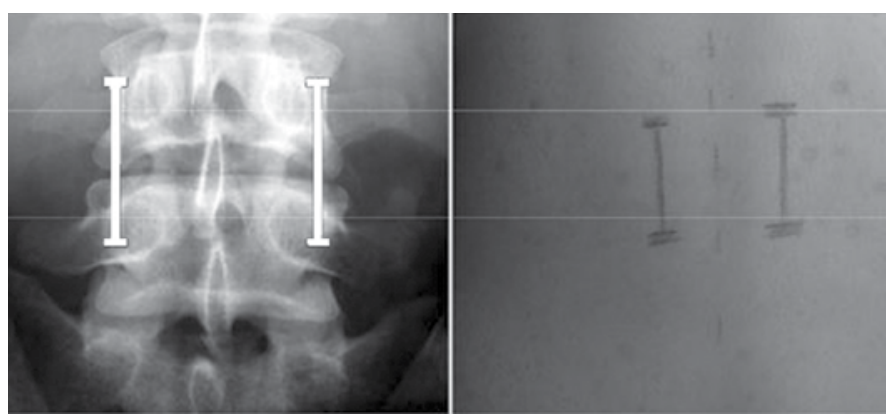

Figura 1. Marcação das incisões na pele.

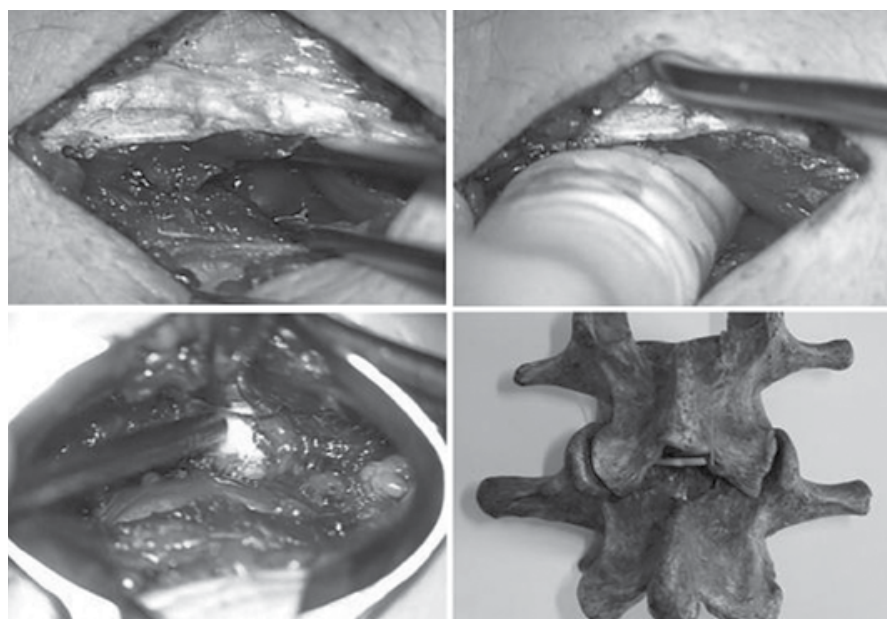

Figura 2. Identificação do plano entre os músculos, dissecção profunda com o dedo e identificação da articulação facetaria. Marcação da zona de ressecção da lâmina e faceta articular. 


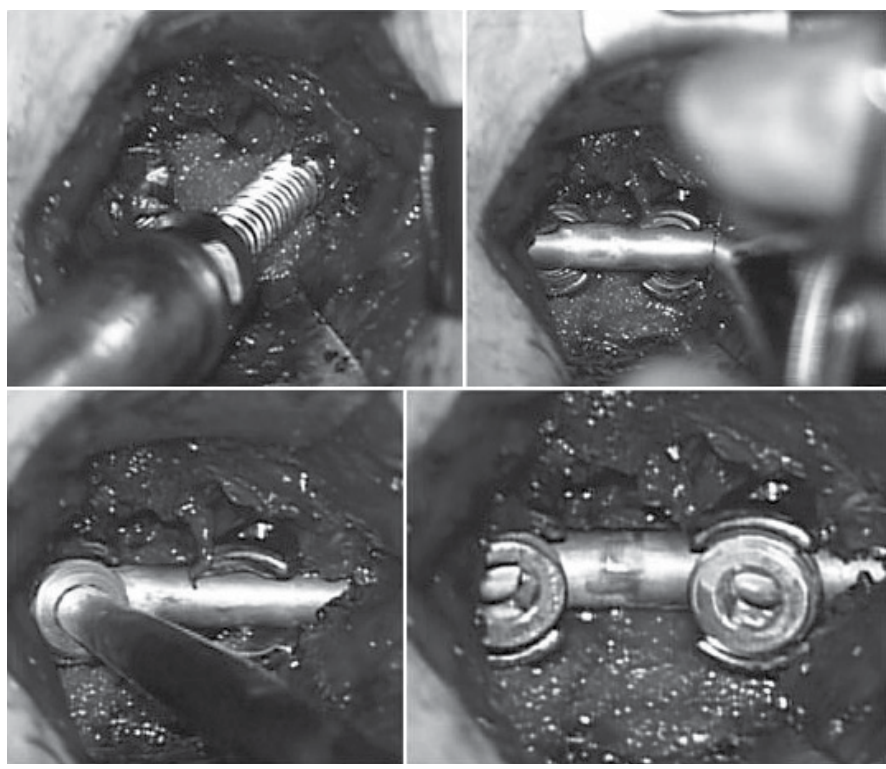

Figura 3. Colocação dos parafusos pediculares.

\section{RESULTADOS}

Os resultados são mostrados resumidamente na Tabela 2. Dos 12 pacientes incluídos no estudo, 6 eram homens e 6 mulheres, com idade média de 38 anos. A indicação mais frequente para o procedimento foi discopatia degenerativa, em 10 casos, sendo 5 casos com e 5 casos sem hérnia discal. Apenas um desses casos tratou-se de uma reabordagem tardia por discopatia severa com recidiva de hérnia, desenvolvida anos depois de uma cirurgia de hérnia discal (Figura 4). Em 2 pacientes a indicação foi espondilolistese espondilolítica com degeneração discal. O nível mais acometido foi L5-S1 (58\%), seguido por L4-L5 (33\%). Em um paciente o nível foi entre L5 e uma vértebra de transição (L5-VT).

Tabela 2. Resumo dos resultados da série.

\begin{tabular}{c|c|c|c|c|c|c}
\hline Paciente & $\begin{array}{c}\text { Tempo de } \\
\text { internação } \\
\text { pós-op. }\end{array}$ & $\begin{array}{c}\text { Oswestry } \\
\text { pré-op. }\end{array}$ & $\begin{array}{c}\text { Oswestry 6 } \\
\text { meses pós-op. }\end{array}$ & $\begin{array}{c}\text { VAS pré- } \\
\text { operatório }\end{array}$ & $\begin{array}{c}\text { VAS 6 } \\
\text { meses P0 }\end{array}$ & $\begin{array}{c}\text { Retorno ao } \\
\text { trabalho }\end{array}$ \\
\hline 1 & 3 dias & $38 \%$ & $16 \%$ & 5 & 2 & Sim \\
\hline 2 & 3 dias & $22 \%$ & $8 \%$ & 4 & 1 & Sim \\
\hline 3 & 4 dias & $24 \%$ & $16 \%$ & 3 & 2 & Sim \\
\hline 4 & 4 dias & $46 \%$ & $18 \%$ & 8 & 3 & Não \\
\hline 5 & 4 dias & $70 \%$ & $32 \%$ & 9 & 3 & Sim* \\
\hline 6 & 3 dias & $24 \%$ & $16 \%$ & 5 & 2 & Sim \\
\hline 7 & 5 dias & $51 \%$ & $44 \%$ & 7 & 8 & Não \\
\hline 8 & 4 dias & $81 \%$ & $20 \%$ & 9 & 1 & Não \\
\hline 9 & 4 dias & $51 \%$ & $26 \%$ & 7 & 3 & Não \\
\hline 10 & 5 dias & $75 \%$ & $35 \%$ & 10 & 3 & Sim \\
\hline 11 & 3 dias & $74 \%$ & $18 \%$ & 9 & 1 & Não \\
\hline 12 & 15 dias** & $46 \%$ & $23 \%$ & 6 & 2 & Não \\
\hline Médias & 4 dias & 50,1 & 22,7 & 6,25 & 2,58 & $50 \%$ \\
\hline
\end{tabular}

* Retornou ao trabalho com função diferente da anterior

**Necessitou de reoperação para reposicionamento de implante.

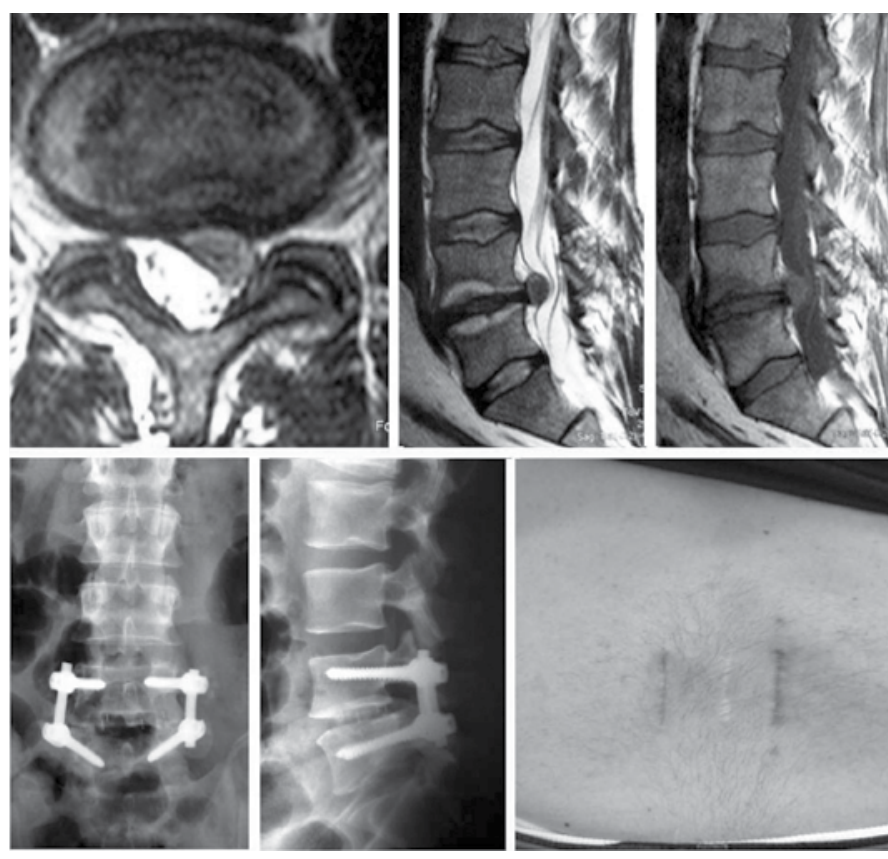

Figura 4. Discopatia grave e recidiva de hérnia discal 4 anos após microdiscectomia prévia. Resultado radiológico e estético após 3 meses.

Em nenhum dos pacientes foi necessário ampliar a abordagem ou converter para a técnica conven sional, de abordagem pela linha média. $O$ tempo cirúrgico variou de 1 hora e 45 minutos a 3 horas e meia, sendo clara a redução do ter po com a melhora da curva de aprendizagem. Em um caso foi necessária reabordagem no quarto dia pós-operatório, para reposicionamento de um parafuso colocado muito medialmente, comprimindo a raiz nervosa, significando um índice de mal posicionamento de implantes de $2 \%$. O tempo de internação pós-operatória foi de 4 dias em média, excluindo-se o paciente que necessitou o reposiciınamento do implante e permaneceu 15 dias internado.

A média do Índice de Oswestry iantes da cirurgia foi de 50,1 pontos percentuais, com variância entre: 22 e 75 pontos percentuais. Já a média do Índice na avaliação corา 6 meses de pós-operatório foi de 22,7 pontos percentuais, com váıriância entre 8 e 44 pontos percentuais. Isso significou uma melhora média do índice de Oswestry 6 meses após a cirurgia de 27,5 pontc s percentuais. As melhoras mais destacadas foram nos casos em quis havia ciatalgia por hérnia discal associada à discopatia, com melhore média de 37 pontos percentuais.

Em relação à escala visual ana ógica de dor (VAS) a média do grupo era de 6,25 antes da cirurgia e de 2,58 aos 6 meses depois da artrodese, significando uma melhora média de 3,6 pontos. Em um caso houve piora no VAS no pós-operatório de 6 meses, de 7 para 8 pontos, mas este mesmo paciente apresentou melhora quando avaliado pelo Índice de Oswestry.

Analisando separadamente as seções de perguntas do Índice de Oswestry, nota-se que os quesitos que apresentaram melhora acentuada foram: a intensidade da dor e a qualidade do sono, enquanto os que apresentaram pior resultado foram:a capacidade de levantamento de pesos e dor ao sentar.

A média de retorno ao trabalho foi de $50 \%$, porém, na série apresentada, apenas 5 pacientes tinham profissão envolvida com trabalho braçal e, destes, apenas 1 (20\%) retornou ao trabalho. Dos 7 pacientes que não tinham ocupação braçal, 5 (70\%) retornaram ao trabalho, sendo que 1 paciente mudou de profissão.

\section{DISCUSSÃO}

Inicialmente aplicada apenas no tratamento de lesões traumáticas, deformidades e outras condições associadas com instabilidade maior, nas últimas décadas a artrodese da coluna lombar 
ganhou um grande espaço também no arsenal de manejo das doenças degenerativas. Um dos fatores que mais contribuiu para isso foi o desenvolvimento, na década de 90, dos cilindros rosqueados de fusão intersomática, pois esses implantes permitiam cirurgias menos agressivas e mais focalizadas na lesão ${ }^{4}$. Em outras palavras, pode-se dizer que a introdução da fusão espinhal no contexto degenerativo se deveu ao desenvolvimento de uma técnica pouco invasiva.

Com o tempo, observou-se que os cilindros rosqueados apresentavam índices altos de pseudartrose, mas a idéia da artrodese na patologia degenerativa já estava bastante disseminada e soava muito mais natural. Os cilindros de fusão foram abandonados e substituídos por técnicas cirúrgicas mais agressivas que, porém, traziam maior estabilidade e melhores índices de sucesso da fusão ${ }^{5}$.

Atualmente o padrão ouro é a artrodese intersomática por via posterior acompanhada de fixação com parafusos pediculares, ou artrodese circunferencial. Esta técnica, quando realizada pela abordagem mediana convencional, pressupõe dissecção ampla e grande retração lateral da musculatura paravertebral, sendo uma cirurgia extremamente agressiva e de morbidade significativa ${ }^{3,6}$.

Nas artrodeses lombares abertas convencionais, a maior parte da morbidade está relacionada ao dano dos tecidos moles e musculatura paraespinhal. Grandes incisões, exposição, dissecção ampla, descolamento e retração muscular por tempo prolongado levam à isquemia e denervação muscular que, por sua vez, resultam em atrofia e dor ${ }^{7,8}$

O multifidus é um músculo que se origina no processo espinhoso lombar e se divide em vários fascículos, com inserção nas facetas articulares dos níveis inferiores, de modo que o músculo nasce em uma vértebra mas se insere em múltiplos segmentos, o que permite a realização de movimentos coordenados complexos de extensão, rotação e inclinação lombar, além de funcionar como um importante elemento de propriocepção e estabilização dinâmica do segmento dorsolombar ${ }^{9}$. Em uma abordagem espinhal mediana, o multifidus pode ser desinserido e reinserido na apófise espinhosa, mas seu suprimento neurovascular só é preservado se o descolamento muscular não se estender lateralmente às facetas articulares, o que não é o caso nas artrodeses de coluna, onde a exposição é feita até os processos transversos ${ }^{9}$.

O multifidus, especificamente, é o maior estabilizador muscular da junção lombosacra, e o comprometimento de sua função, por desinserção ou necrose muscular, pode resultar em perda de estabilidade e distúrbio do movimento local ${ }^{9}$. Essas disfunções do multifidus podem contribuir para a "fusion disease", ou seja, pacientes com sucesso radiológico na artrodese, mas com resultado clínico pobre?.

Recentemente, várias técnicas tem sido desenvolvidas para tentar tornar o tratamento da patologia degenerativa lombar menos invasivo. De um ponto de vista conceitual, existem dois tipos de cirurgia minimamente invasiva, as cirurgias de objetivo pouco invasivo e as de acesso pouco invasivo ${ }^{10}$.

Nas cirurgias de objetivo pouco invasivo usa-se alternativas de tratamento que não a artrodese, como uma nucleoplastia, que apenas reduz a pressão intradiscal, mantendo a integridade de todas as estruturas, ou as fixações dinâmicas, que preservam as articulações e determinado grau de movimento.

As técnicas de acesso pouco invasivo tem os mesmos objetivos de uma artrodese comum, usando, porém, um caminho até o alvo cirúrgico que cause a menor destruição possível em outros tecidos e estruturas periféricas ${ }^{10}$.

Várias técnicas surgiram no intuito de fazer novamente da artrodese lombar uma cirurgia menos invasiva, como a artrodese intersomática via anterior por mini-acesso (mini-ALIF) e outras. Estes procedimentos sugerem a mesma segurança e eficácia das técnicas convencionais, no entanto, a maioria delas resulta em tipos de estabilização que se afastam do padrão ouro, como o uso de espaçadores stand alone, apresentando resultados de médio e longo prazo com índices maiores de falha da consolidação, sendo que nenhuma dessas técnicas conseguiu uma grande popularização.
O princípio do acesso minimamente invasivo é ser o menos traumático possível, ou seja, ele deve seguir estritamente caminhos anatômicos ou espaços já existentes, causando o mínimo de dano aos tecidos vizinhos. Se o dano não puder ser evitado, ele deve ser reparável e provocar um efeito pouco importante no desfecho do tratamento. Sempre que possível a função dos músculos abdominais e paravertebrais deve ser preservada.

O aspecto mais importante do acesso é a exposição adequada do alvo cirúrgico. A patologia deve ser claramente visível e identificável, e o tratamento não deve ter nenhuma restrição imposta pelo acesso reduzido. As manobras de manipulação da coluna e a colocação de implantes também devem ser possíveis ${ }^{10}$.

As técnicas de acesso paramediano à coluna não são novidade, em 1959 Watkins descreveu o acesso aos processos transversos para fusão posterolateral através do plano fascial entre o sacrospinalis (massa formada pelo multifidus e longissimus) e o quadrado lombar. Wiltse e Hutchinson apud Hoh et al. ${ }^{9}$ descreveram o acesso pelo plano entre o multifidus e o longissimus em 1964, para o tratamento de espondilolisteses.

A elaboração destas técnicas, com a identificação da anatomia microcirúrgica da musculatura posterior, resultou na abordagem intermuscular utilizada atualmente.

O grande mérito da abordagem em discussão é preencher todos os princípios dos acessos minimamente invasivos e converter a artrodese circunferencial, atual padrão ouro, em uma técnica minimamente invasiva, sem comprometer os passos cirúrgicos.

A realização da artrodese circunferencial por via posterior minimamente invasiva tem sido descrita com uso de sistemas de instrumental dedicado, produzidos por diferentes fabricantes. Basicamente, são disponibilizados sistemas baseados em afastadores tubulares com mecanismos de dilatação, ou sistemas de afastadores com múltiplas lâminas, móveis em vários sentidos. É usual que o afastador seja fixado à mesa cirúrgica por um braço articulado e que disponha de um sistema de iluminação por fibra óptica.

Todos esses sistemas tem em comum o fato de serem desenvolvidos para uso com implantes bastante específicos, do mesmo fabricante, e terem um custo bastante elevado, pelo menos para os padrões socioeconômicos brasileiros. O objetivo desse trabalho foi tentar realizar a técnica minimamente invasiva utilizando instrumental cirúrgico e implantes comuns, observando se haveria um grau muito grande de dificuldade, aumento dos riscos, ou comprometimento dos resultados.

É claro que a utilização de sistemas de afastadores de alta tecnologia pode facilitar o procedimento, mas eles não são vitais para a aplicação da técnica minimante invasiva. Com a utilização de afastadores cervicais comuns foi perfeitamente possível expor a faceta articular e a lâmina e, ainda, os processos transversos, quando desejado.

Em nosso ponto de vista, a maior desvantagem do afastador comum é a ausência de um sistema integrado de iluminação. Nem todas as salas cirúrgicas possuíam focos que permitissem uma boa iluminação do campo operatório, de forma que usamos em alguns casos lâmpadas frontais e, em outros, o microscópio cirúrgico para descompressão e exploração do espaço discal. O uso do microscópio cirúrgico foi bastante confortável e nos pareceu adequado inclusive quando da utilização de retratores com sistema de iluminação, uma vez que a magnificação proporcionada permite uma visualização bem melhor dos elementos neurais.

Em termos de técnica cirúrgica, a maior diferença entre o uso dos afastadores comuns e os específicos para acesso mini invasivo é que, nos afastadores comuns, é feita uma abordagem com dissecção entre os ventres musculares do longissimus e multifidus, enquanto com muitos sistemas específicos, especialmente os de retratores tubulares e parafusos canulados, o alvo no espaço discal é marcado e a abordagem é feita através dos ventres musculares, com divulsão das fibras. Esta também é uma maneira de manter a integridade do músculo, mas o conceito de acesso através de vias anatômicas naturais é perdido. Segundo alguns autores ${ }^{9}$, e também em nossa experiência, o uso de afastadores tubulares largos pode resultar em invasão do campo cirúrgico por tecido muscular, herniado 
sob as bordas inferiores do tubo, necessitando cauterização e arrancamento da musculatura para exposição adequada da coluna. Os ajustes de exposição do campo cirúrgico pelas mudanças de posição do tubo durante a cirurgia também podem danificar e romper estruturas musculares. Dessa forma, nos parece que simples retratores laminares colocados entre ventres musculares intactos podem ser mais eficientes e permitir uma visão cirúrgica melhor.

Outra diferença é na colocação dos parafusos pediculares. A maioria dos sistemas dedicados utiliza parafusos canulados e preconiza a técnica de puncionar os pedículos com fios guia e introduzir os parafusos posteriormente. Com uso de material comum, a colocação dos parafusos segue os passos tradicionais, com preparação do orifício e colocação direta do parafuso, sob controle de radioscopia. A visão de campo oferecida pelo afastador cervical convencional é perfeitamente suficiente para que se identifiquem os referenciais anatômicos para colocação dos parafusos.

Manobras de distração e compressão podem ser aplicadas para melhor acesso e visualização do espaço discal. Alguns autores realizam essas manobras de maneira indireta, utilizando um suporte cirúrgico de posicionamento variável, tipo Wilson frame ${ }^{11}$. Em nossa série, realizamos essas manobras da maneira usual, com as chaves de compressão e distração fornecidas com os implantes.

Outra questão importante quando se fala de artrodeses minimamente invasivas é a obtenção de enxerto ósseo. Com a abordagem que utilizamos, o acesso à parte posterior da crista ilíaca, por dissecção interfascial é muito fácil quando a cirurgia aborda o disco L5-S1 e possível na maioria das abordagens de L4-5. Em nossa série, utilizamos enxerto exclusivamente da lâmina em alguns casos. Sempre que o enxerto local nos pareceu de qualidade ruim ou pouca quantidade recorremos ao enxerto ilíaco, o que aconteceu em um terço dos casos.
Os resultados da série mostraram uma melhora media de 3,6 pontos no VAS e 27,5 pontos percentuais no Oswestry, sendo que o FDA define uma variação mínima de 15 pontos no Oswestry como melhora significativa em casos de artrodese lombar ${ }^{12}$.

É possível que a melhora marcada nessa série se deva em parte à alta incidência de ciatalgia como causa principal de incapacidade, uma vez que este sintoma costuma responder ao tratamento cirúrgico melhor que a dor lombar. Nos casos com hérnia discal, o escore médio estava no extremo superior da faixa de deficiência grave da escala de Oswestry (média de 57,4 pontos percentuais, sendo a faixa de deficiência grave de 41 a 60 pontos), e o escore médio pós-operatório estava no extremo superior da faixa de deficiência leve $(20,4$ pontos percentuais, sendo a faixa de deficiência leve de 0 a 20 pontos).

Apenas um paciente apresentou resultado desapontador, com piora clínica após a cirurgia, mas isso não esteve relacionado a complicações do procedimento. Um dos casos da série foi uma reintervenção de uma discetomia prévia, não havendo nenhuma dificuldade especial, além da esperada.

\section{CONCLUSÃO}

Os resultados da série demonstram que a artrodese circunferencial da coluna lombosacra por abordagem minimamente-invasiva trans-muscular pode ser realizada com a utilização de afastadores cirúrgicos normais, disponíveis na maioria dos serviços, e implantes de coluna iguais aos utilizados na técnica convencional, sem prejuízo técnico maior. Entretanto, a utilização de materiais especiais, dedicados especificamente à aplicação desta técnica, pode facilitar o procedimento cirúrgico.

\section{REFÊRENCIAS}

1. German JW, Foley KT. Minimal access surgical techniques in the management of the painful lumbar motion segment. Spine (Phila Pa 1976). 2005;30(16Suppl):S52-9.

2. Foley KT, Gupta SK. Percutaneous pedicle screw fixation of the lumbar spine:preliminary clinical results. J Neurosurg. 2002;97(1 Suppl):7-12.

3. Asghar F, Davis R, Bono CM. Evidence-based review: minimally invasive spine surgery vs. open surgery. In: Vaccaro AR, Bono CM, editors. Minimally invasive spine surgery. New York: Informa Healthcare; 2007. p. 13-8.

4. Ray CD. Threaded titanium cages for lumbar interbody fusions. Spine (Phila Pa 1976). 1997:22(6):667-79.

5. Diedrich $O$, Kraft $C N$, Perlick $L$, Schmitt $O$. [The posterior lumbar interbody fusion with cages (PLIF) and transpedicular stabilization]. Zentralbl Neurochir.2001;62(3):106-13.

6. Foley KT, Holly LT, Schwender JD. Minimally invasive lumbar fusion. Spine (Phila Pa 1976). 2003;28(15 Suppl):S26-35.

7. Kim DY, Lee SH, Chung SK, Lee HY. Comparison of multifidus muscle atrophy and trunk

extension muscle strength: percutaneous versus open pedicle screw fixation.Spine (Phila Pa 1976). 2005;30(1):123-9.

8. Stevens KJ, Spenciner DB, Griffiths KL, Kim KD, Zwienenberg-Lee M, Alamin T et al. Comparison of minimally invasive and conventional open posterolateral lumbar fusion using magnetic resonance imaging and retraction pressure studies. J Spinal Disord Tech. 2006;19(2):77-86.

9. Hoh DJ, Wang MY, Ritland SL. Anatomic features of the paramedian muscle-splitting approaches to the lumbar spine. Neurosurgery. 2010;66(3 Suppl):13-24.

10. Mayer HM. Minimally invasive spine surgery. In Mayer HM, editor. Minimally invasive spine surgery. 2nd ed. Germany: Springer-Verlag; 2006; p.3-7.

11. Cardoso MJ, Rosner MK. Does the Wilson frame assist with optimizing surgical exposure for minimally invasive lumbar fusions? Neurosurg Focus. 2010;28(5):E20.

12. Roland M, Fairbank J. The Roland-Morris Disability Questionnaire and the Oswestry Disability Questionnaire. Spine (Phila Pa 1976). 2000;25(24):3115-24. 